\title{
Thanking Our Reviewers and Editorial Team
}

The work of reviewing is essential to the advancement of research publications and to theory and practice. I am very grateful to the following dedicated individuals who served as reviewers for this special issue of Behavioral Development Bulletin dedicated to "Autism and Other Child Developmental Disorders: Early BehaviorAnalytic Interventions."-Martha Pelaez, Editor

Yvonne Barnes-Holmes

National University of Ireland

Debra Bendell

Fielding Graduate University

Kyle D. Bennett

Florida International

University

Cassie Cao

Fred S. Keller School

Ned Carter

Sveríges Kommuner och

Landsting

Maricel Cigales

Florida International

University

Traci Cihon

University of North Texas

Annela Costa

Florida International

University

Elisa Cruz-Torres

Florida Atlantic University

Jacob Daar

Southern Illinois University,

Carbondale

Claudia Dozier

University of Kansas
Carly Moher Eby

The New England Center for Children

Barbara Figueiredo

University of Minho

Sagun Giri

Pennsylvania State University

Hudson Golino

Universidade Salgado de Oliveira

Douglas Greer

Columbia University

Per Holth

Oslo and Akershus University College

Deirdre Kavanagh

Ghent University

Grainne Kent

National University of Ireland, Maynooth

Christina Marie King

Simmons College

Lynn Kern Koegel

University of California, Santa Barbara

Russell Maguire

Simmons College

Patrice Marie Miller

Salem State University 
Katerina Monlux

University of California, Davis Health System

Heidi Skorge Olaff

Oslo and Akershus University College

Luis Perez-Gonzalez

Universidad de Oviedo

Anna Peturdottir

Texas Christian University

Ruth Anne Rehfeldt

Southern Illinois University

Thomas Reio

Florida International University

Denise Ross

Western Michigan University

William Sharp

Emory University, School of Medicine
Jessica Singer-Dudek

Teachers College, Columbia University

Ian Stewart

National University of Ireland, Galway

Greg Stikeleather

Secular Policy Institute, Washington, DC

Jennifer Symon

California State University, Los Angeles

Thomas Szabo

Florida Institute of Technology

James T. Todd

Eastern Michigan University

Janet Twyman

ABA Technologies

Harry Voulgarakis

Fielding Graduate University

Putting this issue together would not have been possible without the help of our editorial team:

Sarthak Giri

Managing Editor

Behavioral Development Bulletin
Andrew Ferreira

Manuscript Coordinator

American Psychological

Association

Mark Winter

Journal Production Manager

American Psychological Association 